\title{
$-4-$
}

\section{Ab-initio Atomic Scale Study of Nearly Frictionless Surfaces}

\author{
S. Ciraci ${ }^{1}$, T. Yildirim ${ }^{2}$, S. Dag ${ }^{3}$ and O. Gulseren ${ }^{1}$ \\ ${ }^{1}$ Department of Physics, Bilkent University, Ankara 06800, Turkey \\ ${ }^{2}$ NIST Center for Neutron Research, National Institute of Standards and Technology, \\ Gaithersburg, MD 20899, USA \\ ${ }^{3}$ Center of Nanophase Materials Science (CNMS) and Computer Science and \\ Mathematics Division, Oak Ridge National Laboratory, Oak Ridge, TN 37830, USA
}

\subsection{INTRODUCTION}

The dry sliding friction [1-3] of two objects, which are in direct contact through their asperities, involves many interesting and complex phenomena induced by the long- and short-range forces [4,5], such as adhesion, wetting, atom-exchange, bond-breaking and bond-formation, elastic and plastic deformation. In general, phonons are generated and electron-hole pairs are created at the expense of damped mechanical energy. Even the photons can be emitted. The nonequilibrium phonon distribution generated locally is dissipated by phonon-phonon and electron-phonon coupling. Simulations of dry sliding friction between a metal asperity and an incommensurate metal surface have revealed unusual atomic processes [6]. For example, the lateral force exhibits a quasiperiodic variation with the displacement of an asperity; each period consists of two different stick-slip processes involving structural transitions. It has been also found that the perpendicular elastic deformation of the substrate that is induced by the sliding object is crucial in the energy damping in friction [7]. In certain condition, owing to the elastic deformation of the substrate the corrugation of the surface potential energy can be inverted under high loading forces. This situation gives rise to the occurrence of second state (or bistability) in the stick-slip motion and anisotropy in the hysteresis curve [7]. It is also very well-known that the stiffer the sliding surfaces, the smaller is the friction coefficient [7].

The dry sliding friction between atomically flat, commensurate or incommensurate sliding surfaces is perhaps the simplest but most fundamental type of friction in tribology. 
The relative motion of two commensurate surfaces can take place through repeating stickslip stages and hence helps us to visualize the energy damping under weak elastic deformation. However, the situation is rather complex if the sliding surfaces are incommensurate and undergo an elastic or plastic deformation involving the atom-exchange and wear. The atomic-scale analysis of the interaction between sliding surfaces is necessary to understand the nature of non-conservative lateral forces and various mechanisms of energy damping. In fact, studies based on the Tomlinson's model [8] or calculations using Frenkel-Kontorova model [9] have revealed valuable information about atomic processes in friction.

The invention of atomic force microscope [10] and the friction force microscope [11,12] has made an important impact on the science of friction and opened a new field called nanotribology. Nowadays, various atomic processes can be easily observed and lateral forces in the range of a fraction of nano Newton $\left(1 \mathrm{nN}=10^{-9} \mathrm{~N}=0.62415 \mathrm{eV} / \AA\right)$ can be measured with precision by using these microscopes. Meanwhile atomic scale simulations involving several atoms have been performed using realistic empirical potentials [13-18]. Moreover, ab-initio studies based on Density Functional Theory (DFT) [19] treating relatively smaller systems have provided accurate calculations of interaction forces between two surfaces $[5,20]$ and also led to the development of new empirical potentials. Theoretical studies, on the other side, have started to investigate microscopic aspects of energy transfer and energy damping processes [21-24].

While friction invokes the lost of enormous resources, the lowering of the friction coefficient has been the principal goal in various fields of science and technology. Lubricants have been used to lower the friction coefficients and to eliminate the wear in machining and in the transportation industries. In the last decade, progress made in materials science and surface coating technologies has led to a steady lowering of the friction coefficient. Developing of nearly frictionless surfaces or coating materials has been an ultimate goal of tribology and surface physics. In this work we carry out an atomic scale study based on ab-initio (first-principles) calculations and reveal physical mechanisms underlying the superlow friction coefficient in dry sliding friction. To this end, we investigate dry sliding friction between the commensurate surfaces of a covalent crystal (namely friction between two diamond (001) surfaces) and an ionic crystal (namely friction between two BN (001) surface). We examine interaction between bare surfaces and explore the effect of hydrogenation.

The organization of this chapter is as follows: We first present general theoretical arguments regarding to the nearly frictionless sliding friction and outline recent experimental progress made in the field. We review theoretical methods and present a short description for the atomistic model and essential features of our ab-initio calculations. Discussion of our results obtained from the ab-initio calculations on the sliding friction of two diamond (001) and two BN (001) surfaces constitute the prime part of this chapter. 


\subsection{FRICTIONLESS SLIDING}

\subsubsection{General Theoretical Arguments}

Absence of energy damping in mesoscopic objects has been pointed out earlier [25]. This question can be clarified by examining the energy damping agents involved in friction. These are long- and short-range interactions between two surfaces and various elementary excitations, such as phonon, electron-hole creation, charge density waves and photon emission. High energy excitations such as surface plasmons do not contribute to the energy damping process. Bond-breaking or rebonding, and atom exchange between surfaces and local surface reconstruction can damp mechanical energy and or mediate excitations. Normally acoustic phonons with small excitation energy can easily be excited and hence contribute to the energy damping process. Experiments performed using non-contact AFM [26] have shown that the tip vibrating over the sample gives rise to the energy dissipation even if its minimum spacing from the surface is farther than the range of short-range forces [27-30]. This argument eliminates the possibility that absolutely zero coefficient of kinetic friction, namely $\mu_{k}=0$ can ever occur. Apparently, superlubricity with $\mu_{k}=0$ (reminiscent of superconductivity or superfluidity) cannot be achieved in normal operation conditions, but very low (superlow) friction coefficient is a target one can achieve.

The interaction energy, $E_{i}(\rho, z)$ between two flat surfaces is a function of their spacing $z$ and their relative lateral position $\rho=x \hat{i}+y \hat{j}$. Usually, the interaction energy is small and attractive (i.e. $\left.E_{i}<0\right)$ for large $z(z<0)$, but decreases (becomes more attractive) as $z$ decreases. It then starts to increase by passing through a minimum and eventually becomes repulsive (i.e. $E_{i}>0$ ). The attractive interaction energy is specified as adhesion between two surfaces and involves the formation of bonds between surfaces which may give rise to high friction coefficient in the course of sliding motion. Under loading forces $E_{i}$ can increase and change into repulsive range where elastic and at least local plastic deformations may occur. Substances (solid lubricants, inert gas atoms, etc.) are placed between surfaces to weaken $E_{i}$. In ultrahigh vacuum conditions, the friction coefficient as low as $\mu=0.01$ have been observed for $\mathrm{MoS}_{2}$ and diamond-like carbon (DLC) coatings [31-33]. Even if the lubrication of surfaces coated with such low friction coefficient materials appears to be desirable, the low friction coefficient can increase under different ambient and operation conditions. Coating of surfaces with special materials resulting in repulsive interaction for a wide range of spacing between sliding surfaces is required. Then the loading force will be balanced by the repulsive force derived from the interaction energy, $F_{z}(\rho, z)=-\partial E_{i}(\rho, z) / \partial z$ and the atoms on one surface will be prevented from merging into other surface through a large spacing maintained between them. This way bond-breaking, rebonding and severe deformations will be eliminated. Flying of trains over the superconductive rails is reminiscent of the sliding of surfaces under a repulsive interaction between coated surfaces.

In order to reduce the energy damping in the relative motion and hence to lower $\mu_{k}$ one has to also take the force constants determining the vibration frequencies of atoms into account. It is well-known that the stiffer the sliding surface the lower is the friction constant. Being the principal energy damping agents, the availability of phonons which can get excited by the any sudden release of elastic deformation is not favored. In this respect, 
coating materials made of short and stiff surface bonds are required in nearly frictionless sliding.

\subsubsection{Recent Experimental Progress}

In an effort to lower the friction coefficient, Erdemir et al. [34-36] reported superlow friction and wear between diamond-like carbon (DLC) coated surfaces using a hydrogen-rich plasma. They achieved kinetic friction coefficient $\mu_{k}$ as low as 0.001 and wear rates of $10^{-9}-10^{-10} \mathrm{~mm}^{3} / \mathrm{Nm}$ in inert gas environment under $10 \mathrm{~N}$ load and $0.2-0.5 \mathrm{~m} / \mathrm{s}$ sliding velocities. It has been shown that observed low magnitude and time-variation of $\mu_{k}$ have a close correlation with hydrogen content of the source gas. This work by Erdemir and his coworkers has been a breakthrough towards the achievement of superlow friction and long durability of moving parts in various mechanical applications ranging from the automotive industry to nanotechnology.

\subsection{DESCRIPTION OF THEORETICAL MODEL}

Normally, the structure of sliding surfaces contains several types of defects, such as asperities of different shapes and sizes, vacancies, impurities, domains, etc. A realistic simulation of dry sliding friction has to include all these defects. Hence the atomistic models of sample surfaces have to comprise a large number of atoms. In this respect, the classical molecular dynamics (CMD) method using empirical potentials to represent atomic scale interaction is convenient for the simulation of friction process. Recently, various processes have been simulated and new structures have been predicted by using empirical potentials developed for certain systems. Since numerical calculations using these empirical potentials are not time-consuming, large systems comprising several thousands of atoms have been treated. However, the main drawback of CMD simulations appears when a completely new system is treated. In particular, when the character of the surface atoms (such as their effective charge and bonds) deviate dramatically from those of bulk structure. Under these circumstances, whether the empirical potential parameterized using the bulk properties becomes questionable. On the other side, ab-initio calculations can provide reliable results for the optimized atomic structure, mechanical, electronic and magnetic properties and phonon density of states of a given system, if it involves small number (200-300) of atoms. Various mechanisms underlying the energy dissipation and estimations of friction coefficient with upper and lower limits can be revealed. In this respect, ab-initio methods are superior to classical methods if the system can be represented by 200-300 atoms. Besides, ab-initio methods are complementary to CMD in revealing the correct charge and bonding structure and hence in developing reliable empirical potentials.

\subsubsection{Atomistic Models and Details for Ab-initio Calculations}

In this study, sliding surfaces are represented by two infinite slabs made from the atomic layers of the coating materials. The atoms of slabs are treated in two different categories. In the first category, the atoms at the back surfaces of both slabs are kept fixed in their ideal 
configurations, $x_{i}, y_{i}, z_{i}$. The layers of fixed atoms represent sample or coating layers away from the sliding surface. Normally, they are not affected from the friction process. By displacing all the fixed atoms of one slab relative to the fixed atoms of the other slab one can achieve a lateral displacement of two slabs and induce a loading force. The atoms at the surface region of the slabs facing each other are treated in the second category and are relaxed while the first category atoms are fixed at given $x_{i}, y_{i}, z_{i}$ positions. This way, relative sliding of two slabs including atomic displacements, elastic and plastic deformations, etc. are modeled from the first-principles.

The atomic process and forces in sliding friction have been investigated by carrying out calculations from the first-principles within DFT, which were proven to yield accurate predictions for many metal and insulator surfaces. Here we present crucial parameters of first-principle calculations. The sliding friction is treated either by supercell method using periodic boundary conditions or by finite size surfaces using local basis set. In the supercell method, the wave functions are expressed in momentum space. The magnitude of the largest wave vector sets the cutoff energy and hence the number of plane waves used in the expansion. The ionic potentials are represented by ultrasoft pseudopotentials $[37,38]$ and then the cutoff energy is taken $300 \mathrm{eV}$. The exchange correlation potential is represented by using Generalized Gradient Approximation [39]. The Brillouin zone corresponding to the supercell is sampled within the Monkhorst-Pack special $k$-point scheme [40]. Optimization of atomic positions is performed by conjugate gradient method. Lateral components $F_{x}, F_{y}$ and perpendicular component $F_{z}$ of the net force induced between two slabs are calculated. We did not included the long-range Van der Waals force since it is negligible as compared to the perpendicular force $F_{z}$ induced under high loading force $F_{N}$.

\subsection{SUPERLOW FRICTION COEFFICIENT BETWEEN HYDROGENATED DIAMOND SURFACES}

Hydrogenated DLC (H:DLC) coating by all means is a complex, amorphous structure showing various irregularities. The sliding surfaces cannot be commensurate and contain irregularly distributed asperities and perhaps voids. We believe that determination of the structure of DLC by itself is important and treated earlier [42]. However, even if the structure of H:DLC realized in superlow friction [34-36] and also physical and chemical processes taking place in the course of friction are stochastic in nature, the local bond orders and $\mathrm{C}-\mathrm{H}$ bond topology are expected to be similar to various hydrogenated diamond surfaces. Therefore, the interaction between H:DLC surfaces and the nature of interaction between these surfaces can be understood by using two hydrogenated diamond surfaces. In this section we will present our study of superlow friction coefficient between two hydrogenated diamond $(001)-(2 \times 1)$ surfaces using first-principle plane wave method [41]. Clearly, our study does not promise a realistic simulation of the experiment resulted with superlow friction coefficient [34]. Our objective in this atomic scale study is to better understand the physical mechanisms involved in the superlow friction observed between hydrogenated DLC coated surfaces [34]. We hope that the ingredients of the superlow friction coefficient revealed from our study will be useful for developing new coating materials 
which are stable in the desired operating conditions. In particular, developing coating materials which can sustain to ambient conditions and oxidation is the objective our study.

How simple our model can be, its two features are of particular importance. These are the full relaxation of surface atoms at any instant of sliding process, and accurate calculation of the variation of lateral force components under the constant loading force $F_{N}$. Diamond $(001)-(2 \times 1)$ surfaces are represented by two slabs facing each other at a distance. Each slab consists of 6 layers of carbon atoms. Carbon atoms at the back surface of each slab are saturated by hydrogen atoms. The atomic structure of the one individual slab is first optimized and then the carbon atoms at the 6th layer (at the back surface of the slab) and saturating $\mathrm{H}$ atoms (i.e. those atoms in the first category) are kept at their equilibrium positions. We believe that such configuration mimics the semi-infinite slab (or thick coating).

Figure 4.1(a) illustrates two diamond (001)- $(2 \times 1)$ slab with $\mathrm{H}$ saturated, fixed back sides. The other surfaces of slabs face each other and are free when the distance $d$ between them is large. The structural parameters of the bare surface which reconstructs to form dimer bonds are successfully reproduced.

The normal force $F_{z}$ originate from the short-range interaction between the surfaces of the slab. To this end, we kept the distance $D$ between the back surfaces of the slabs at the preset value and calculated the total energy of whole system, $E_{T}(D, \rho)$ and total force on one of the slabs. Here the total energy and total force are obtained after the optimization of positions of atoms in the second category. We note that since two slabs are pressed against each other by fixing $D$, the calculated forces on the atoms at the back side balance the external (loading) forces which maintain $D$ at a preset value. Therefore, total calculated

(a)

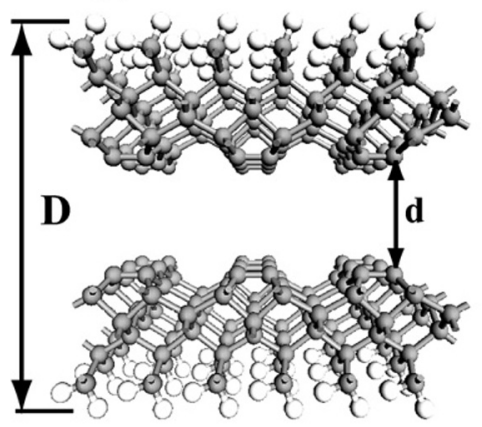

(b)

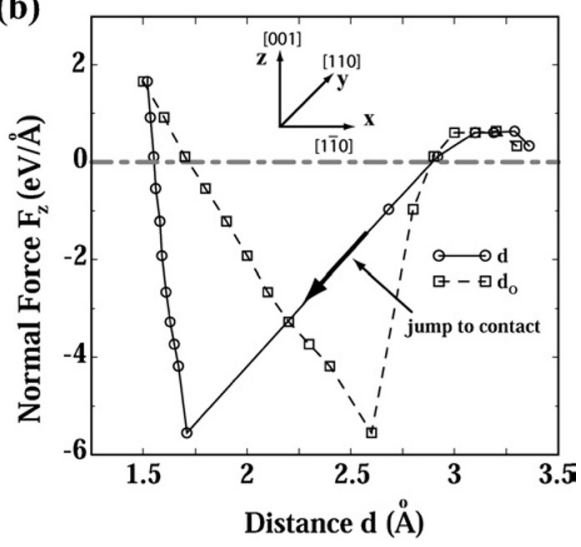

Figure 4.1 (a) Two diamond $(001)-(2 \times 1)$ slabs used to model the sliding of two diamond (001) surfaces. Carbon atoms at the back sides of the slabs are saturated by $\mathrm{H}$ atoms. The positions of these carbon atoms and those of saturating $\mathrm{H}$ atoms are fixed at the configuration corresponding to that obtained from the optimization of individual (free) slabs. The distance between the back surfaces of slabs is $D$, and that between two sliding surfaces facing each other is $d$. The crystal directions are identified by Cartesian axes shown by inset. $\mathrm{C}$ and $\mathrm{H}$ atoms are shown by filled and empty spheres, respectively. (b) Calculated normal force $F_{z}$ is generated when two diamond (001) slabs are pressed towards each other by decreasing $D$ and hence $d . d_{o}$ and $d$ correspond to spacing between two sliding diamond $(001)-(2 \times 1)$ surfaces before and after relaxation. (Reproduced from [41].) 
vertical force on one of the slabs is equal to the vertical interaction force $F_{z}$. By definition the loading force $F_{N}=-F_{z}$. The variation of $F_{z}$ is plotted in Figure 4.1(b) with respect to the separation between slab surfaces before relaxation, $d_{o}$, as well as the actual separation, $d$ after the relaxation. The interaction is weak and repulsive for $d>2.75 \AA$, but $F_{z}$ becomes attractive as $D$ decreases and then jumps to contact attaining the value approximately equal to $-6 \mathrm{eV} / \AA$. Strong bonds form between the sliding surfaces of two diamond (001) $-(2 \times 1)$ slabs near equilibrium separation corresponding to $F_{z} \simeq 0$. Once a normal force is applied to press the slabs against each other, atoms of different surfaces come close to each other at $d \sim 1.5 \AA$ and subsequently $F_{z}$ becomes repulsive. Under these circumstances, since the sliding motion can involve local deformations, bond-breaking and rebonding, the dynamical friction coefficient, $\mu_{k}$ as well as the wear rate are expected to be high. In fact, the dynamical friction coefficient has been measured to be equal to 0.65 for the sliding DLC-coated surfaces which are free of hydrogen.

\subsubsection{Force Variations in the Sliding Friction of Two Hydrogenated Diamond Surfaces}

First, we will examine the variations of normal force when the sliding diamond surfaces are hydrogenated. Dangling bonds of carbon atoms on two slab surfaces facing each other are saturated by $\mathrm{H}$ atoms to form a monohydride phase, i.e. H:diamond $(001)-(2 \times 1)$. Upon the saturation of the surface dangling bonds the dangling bond surface states disappear and a wide energy gap opens between the valence and conduction bands of the slab. The surface charge density differs dramatically from that of the clean diamond $(001)-(2 \times 1)$. In Figure 4.2 we show the atomic configuration of the H:diamond $(001)-(2 \times 1)$ surfaces.

Moreover, Mulliken analysis indicates that 0.25 electrons are transferred from $\mathrm{H}$ atom to the $\mathrm{C}$ atom that is bound to it. This situations complies with the fact that the $\mathrm{C}$ atom is more electronegative than the $\mathrm{H}$ atom. As a result, the $\mathrm{H}$ atom is positively charged. The depletion of electrons on $\mathrm{H}$ atoms induces a repulsive interaction and hence a repulsive $F_{z}$ even for $d<2.5 \AA$ between H:diamond (001)- $(2 \times 1)$ surfaces. This is the most essential feature in obtaining superlow friction coefficient from H:DLC coated sliding surfaces. The variation of $F_{z}$ with spacing $d$ is shown in Figure 4.2. This repulsive force $F_{z}$ keeps the sliding surfaces wide apart at a distance $d$ and balances the loading force $F_{N}$. As a result, sliding surfaces are prevented from being too close. This way, deformation of $\mathrm{C}-\mathrm{H}$ bonds are suppressed to a great extend. It is interesting to note that like carbon silicon is also a Group IV element and has diamond structure. A strong attractive interaction is generated between clean Si (001) slabs. However, similar to diamond (001) slabs, the attractive interaction turns repulsive upon the hydrogenation of $\mathrm{Si}$ (001) surfaces and generates a strong repulsive normal force. It appears that $\mathrm{H}: \mathrm{Si}(001)-(2 \times 1)$ displays a feature similar to that of H:diamond $(001)-(2 \times 1)$. We next examine whether this feature, namely the repulsive normal force between surfaces can lead to superlow friction coefficient.

\subsubsection{Sliding Friction of Hydrogenated Diamond (001) Slabs}

Having examined the perpendicular variation of $F_{z}$, we now address following questions: (i) Does the repulsive interaction continue to keep surfaces wide apart, if one of the diamond slabs is laterally displaced relative to other one. (ii) What is the range of $F_{N}$ 
(a)

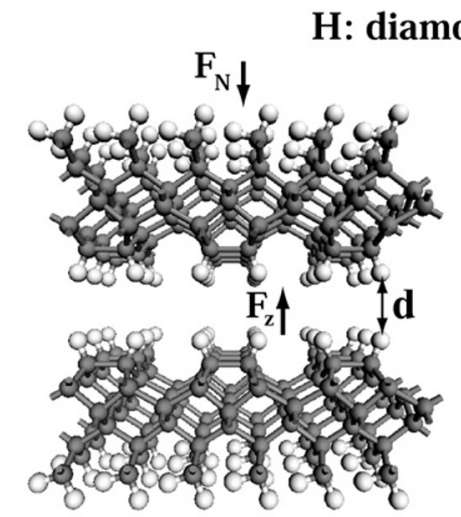

(b)
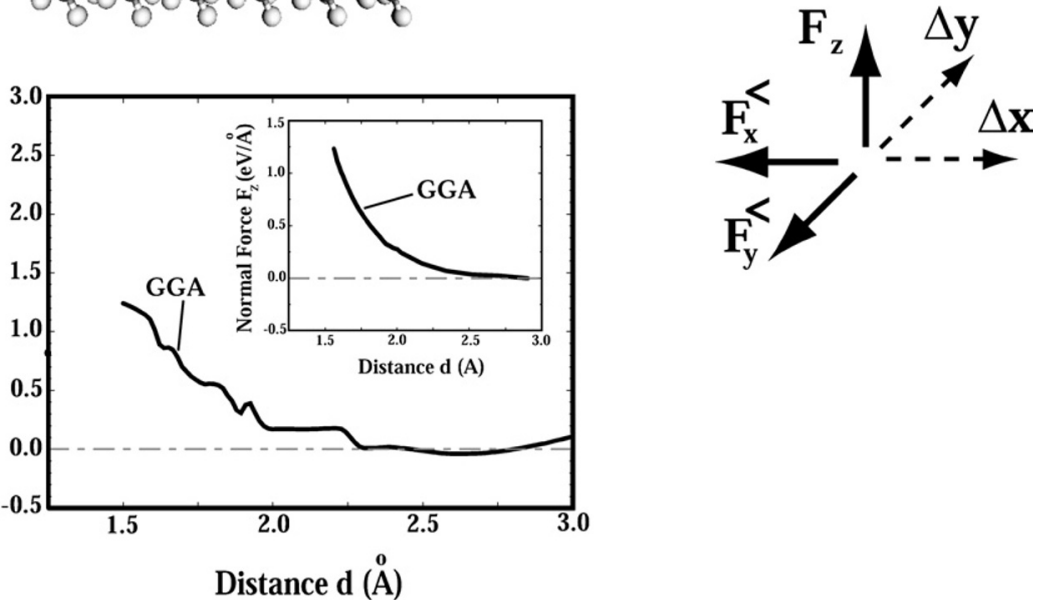

Figure 4.2 (a) Atomic configuration of two diamond $(001)-(2 \times 1)$ slabs with the dangling bonds on the surfaces facing each other are saturated by hydrogen atoms to form monohydride phase H:diamond $(001)-(2 \times 1)$. The spacing $d$ between these surfaces, normal force $F_{z}$, loading force $F_{N}$. (b) Variation of the calculated normal force $F_{z}$ between the two surfaces of $\mathrm{H}$ :diamond $(001)-(2 \times 1)$ as a function of their actual, relaxed separation $d . F_{z}$ is generated when two diamond (001) slabs are pressed towards each other by decreasing $D$. The inset shows the variation of the same force between hydrogenated Si $(001)-(2 \times 1)$ surfaces. Right: Directions of the loading force $F_{N}, F_{z}$, lateral force components $F_{x, y}$, and lateral displacements, $\Delta x$ and $\Delta y$. Lateral force component, which is in opposite direction of displacement is indicated by superscript " $<$ ". (Reproduced from [41].)

where the repulsive interaction between surfaces persists without any serious deformation? (iii) Can one present an upper limit for the friction coefficient? To answer all these questions we carried out a series of first-principle calculations for the interaction energy $E_{i}$, normal force $F_{z}$, lateral force $F_{L}$ corresponding to different loading force (hence $D$ ) and displacements $(\Delta x, \Delta y)$ of the upper slab. In these calculations, all the atoms have been relaxed, except $\mathrm{C}$ and $\mathrm{H}$ atoms at the back side of both slabs. The latter atoms are kept fixed in their ideal configurations after their planes are displaced to different perpendicular and lateral positions by varying $D$ and $(\Delta x, \Delta y)$ in sequential increments. We note that by keeping two back ends of slabs at a distance $D$ but relaxing the rest of the atoms induces a loading force $F_{N}(D)$, which in turn is balanced by $F_{z} . F_{z}$ itself is obtained from the sum 


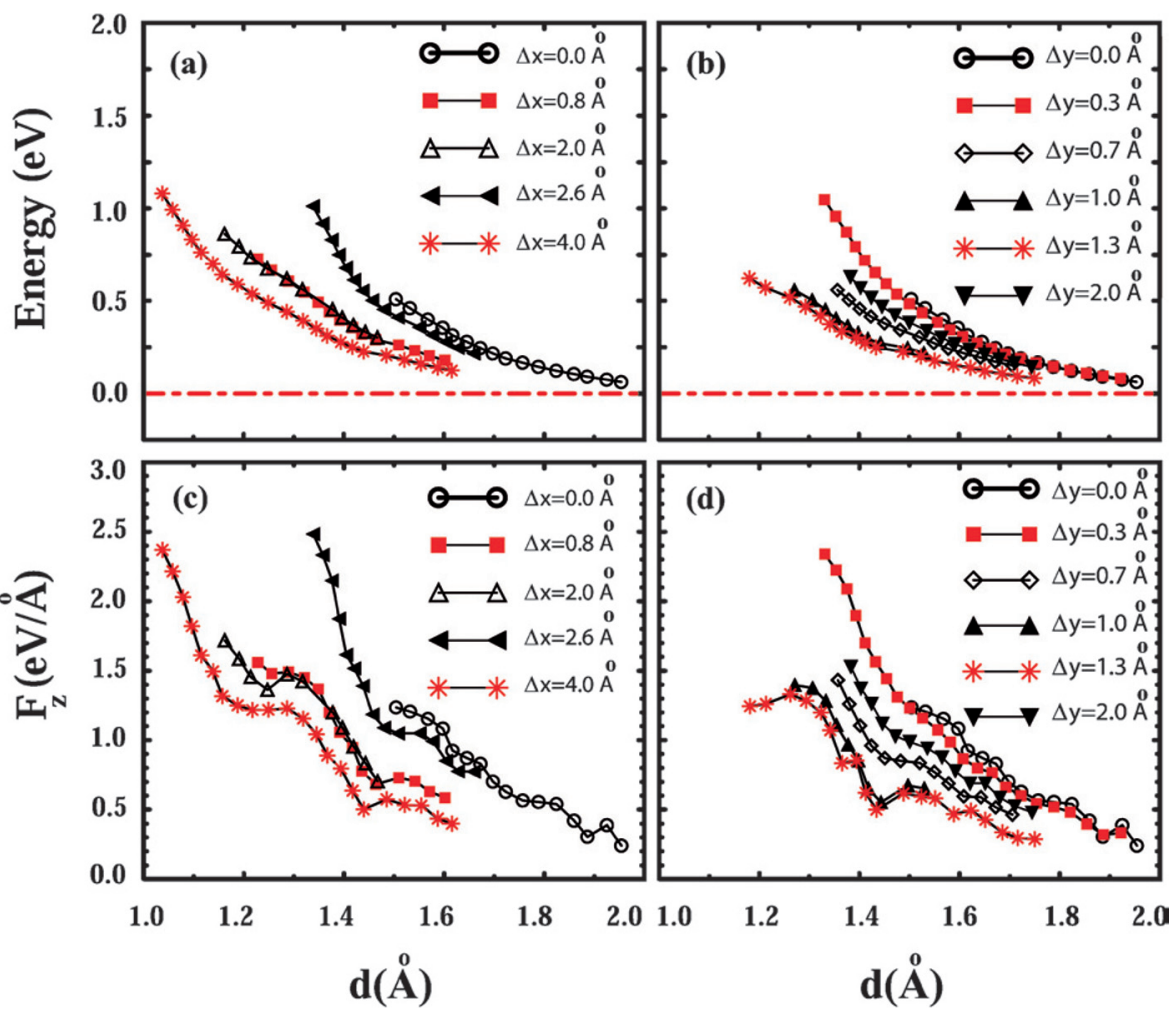

Figure 4.3 (a) and (b) Variation of the calculated interaction energy $E_{i}$ as a function of the perpendicular distance $d$ calculated at the different lateral displacements $\Delta x$ and $\Delta y$. (c) and (d) The same variation of the normal force $F_{z}$. Energy and force units correspond to per $(2 \times 1)$ unit cell. (Reproduced from [41].)

of the perpendicular component of calculated forces on the fixed atoms of one of the slabs. Namely $F_{z}=\sum_{i} F_{z, i}$, where $i$ is the index of fixed atoms of one slab. The same sum on the other slab yields $F_{z}$ with the same magnitude but in opposite direction. Similarly, the lateral forces along $x$ (or $y$ ) axis are obtained from the sum $F_{x(y)}=\sum_{i} F_{i, x(y)}$. Performing $a b$-initio calculations of $E_{i}, F_{z}, F_{x, y}$ as a function of $d$ (or $D$ ) at different relative lateral positions, $\Delta x$ and $\Delta y$ a data base is created. In these calculations, the values of $D$ have been varied in small steps to yield normal forces in an appropriate range of interest.

Figure 4.3 presents calculated variations of $E_{i}$ and $F_{z}$ as a function of $d$ for different lateral displacements, $\Delta x$ and $\Delta y$, of the top slab. Here we note that the interaction energy $E_{i}=E_{T}-E_{T, d=\infty}$, where $E_{T, d=\infty}$ is the total energy corresponding to very large $d$ (or twice the total energy of one slab in the absence of the other one). We note that the variation of $E_{i}$ and $F_{z}$ is not a smooth function owing to discrete changes of $D$ and to the relaxation of the $\mathrm{C}-\mathrm{H}$ bonds. Note that since $F_{z}$ remains always repulsive and strong even at significantly large spacing, the sliding surfaces are kept apart even for large loading forces. As a result, $\mathrm{C}-\mathrm{H}$ bonds of different surfaces neither merge nor interfere with each other. In the course of sliding $\mathrm{C}-\mathrm{H}$ bonds experience neither significant deformation (i.e. bending, stretching or shrinking) nor wear through bonding-rebonding. 
If the sliding motion were adiabatic, no energy would be damped in sliding motion of two commensurate surfaces like we treat here. However, this is not the case; various sudden processes generate excitations and give rise to energy damping. Now we present a global approach to estimate an upper limit for $\mu_{k}$ using the variation of the lateral force from the present calculations. To this end we consider displacements along $x$ - and $y$-axis and we construct lateral force variation under the given constant loading force using our data base, namely $F_{L=x}\left(\Delta x, \Delta y=0, F_{N}\right)$ and $F_{L=y}\left(\Delta x=0, \Delta y, F_{N}\right)$. Keeping the loading force $F_{N}$ constant is the most difficult part of our study and requires a large number of numerical calculations corresponding to different $\Delta x, \Delta y$ and $D$. We considered the loading force $F_{N}=1$ and $1.2 \mathrm{eV} / \AA$ per cell, which are actually too high as compared to the loading force in the experiment [34] as well as in practical applications. In this respect, our estimation of $\mu_{k}$ is a stringent test. The variations of $F_{x}$ and $F_{y}$ are illustrated in Figure 4.4. For the reasons pointed out earlier the variation of lateral force is not smooth. Since lateral force is calculated using coarse displacement steps of $D$, the elastic deformation of slabs and $\mathrm{C}-\mathrm{H}$ bonds induced by sliding are released suddenly. This gives rise to the stick-slip process as described by the Tomlinson's model [8]. Of course, error bars involved in the calculation of forces. In particular, achieving the constraint of the constant loading force by limited number of data in the data base generated through ab-initio calculations can lead to hysteric variation of the lateral force. Now as an ad hoc approach to estimate $\mu_{k}$ in an energy damping medium, we assume that the work done by the lateral force $F_{L}$ (i.e. when it is parallel to the direction of motion as denoted by $F_{L}^{>}$) is totally lost. Then the average friction force is calculated by $\bar{F}_{f}=\int F_{x}^{<} \mathrm{d} x / R, R$ being the period of the motion.

Using the data in Figure 4.4 we extract the average friction force, $\bar{F}_{f} \sim 0.05 \mathrm{eV} / \AA$ for $F_{N}=1 \mathrm{eV} / \AA$ and $\bar{F}_{f} \sim 0.07 \mathrm{eV} / \AA$ for $F_{N}=1.2 \mathrm{eV} / \AA$. Then the kinetic friction coefficient is calculated from $\mu_{k}=\bar{F}_{f} / F_{N}$ to be approximately 0.05 for both cases. A more realistic estimation could be obtained from $\bar{F}_{f}=\int\left(F_{x}^{<}+F_{x}^{>}\right) \mathrm{d} x / R$ if lateral force variation were calculated precisely. Although our force variations in Figure 4.4 are too crude to obtain precise values, $\mu_{k}$ is calculated for the sake of comparison to be $\sim 0.01$.
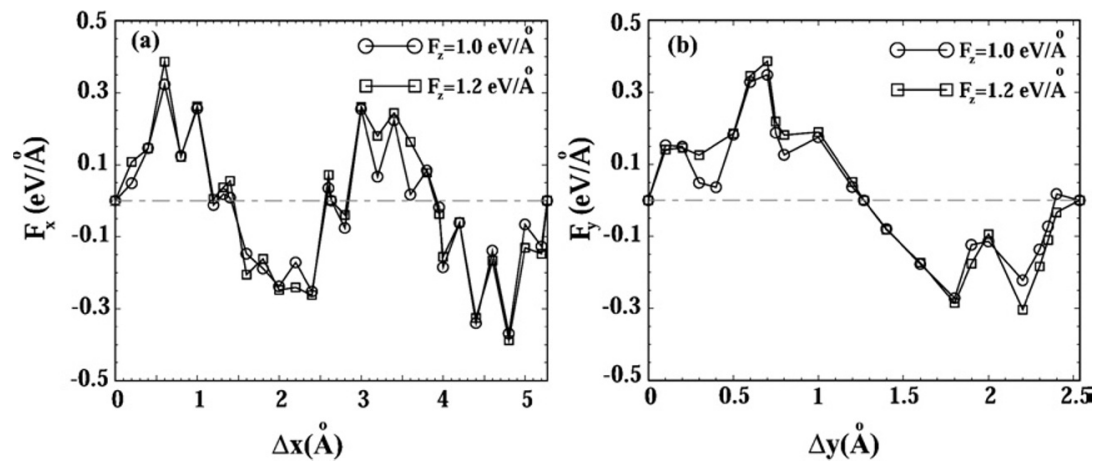

Figure 4.4 (a) Variation of the lateral force, $F_{L=x}$ [in $\mathrm{eV} / \AA$ per $(2 \times 1)$ cell] as a function of the displacement $\Delta x$ of the top slab relative to the bottom one. (b) Same as (a) for the displacement $\Delta y$. In the course of sliding the loading force $F_{N}$ is taken approximately constant. (Reproduced from [41].) 


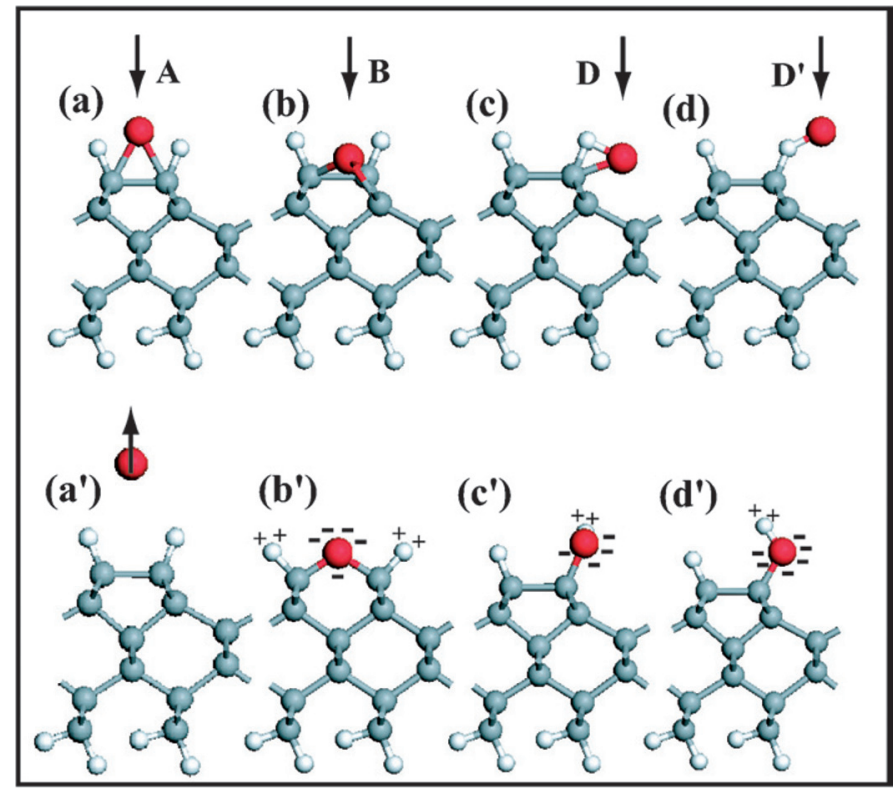

Figure 4.5 Calculated atomic configurations showing the effect of the oxygen atom on the H:diamond $(001)-(2 \times 1)$ surface. (a)-(d) The oxygen atom is placed at different sites of the surface unit cell before the relaxation of the surface. $\left(\mathrm{a}^{\prime}\right)-\left(\mathrm{d}^{\prime}\right)$ Atomic structure and bonding after relaxation of the system. The adsorbed oxygen atom is negatively charged. (Reproduced from [41].)

It should be noted that in the sliding of commensurate surfaces the lateral forces acting on each atom or cell are added constructively to yield a high total lateral force. These lateral forces are, however, conservative and do not give rise to energy damping, if the sliding motion is adiabatic. In the case of incommensurate surfaces, the total lateral force is relatively lower owing to the cancellations. H:DLC coated surfaces can be viewed incommensurate except that the disorder gives rise to higher energy damping. Consequently, the above estimation of $\mu_{k}$ obtained from hydrogenated diamond surfaces with the assumption that all mechanical energy stored into elastic is damped, is an upper limit for H:DLC coated surfaces; but it is still too low.

\subsubsection{Effect of Oxidation}

That the superlow friction coefficient obtained from H:DLC coated surfaces cannot be sustainable in the ambient conditions is the most serious issue [34,35]. The oxygen atom is the potential candidate which destroys the superlow friction when H:DLC coating is exposed to the air. In what follows, we clarify the effect of oxygen on the hydrogenated DLC coating leading to superlow friction. To test the effect of oxygen, we placed $\mathrm{O}$ atoms at the proximity of different sites of the H:diamond $(001)-(2 \times 1)$ surface. Upon relaxation, the system attains the minimum energy configuration, whereby $\mathrm{O}$ atoms break the surface bonds to form new $\mathrm{C}-\mathrm{O}-\mathrm{C}$, or $\mathrm{C}-\mathrm{O}-\mathrm{H}$ and $\mathrm{C}-\mathrm{O}$ bonds and hence they become attached to the surface. Favorably, they attacked the $\mathrm{C}-\mathrm{H}$ bonds to form $\mathrm{C}-\mathrm{O}-\mathrm{H}$ radicals. Charge 
transferred to $\mathrm{O}$ from $\mathrm{H}$ and $\mathrm{C}$ makes $\mathrm{O}$ atom negatively charged as shown in Figure 4.5. This way the interaction between two atoms in different surfaces can be attractive when they carry charge of different polarity. As a result, the steady and strong repulsive interaction between the H:diamond $(001)-(2 \times 1)$ surfaces gradually becomes weaker or turns attractive. Eventually, the superlow friction ends.

\subsection{AB-INITIO STUDY OF ATOMIC-SCALE FRICTION BETWEEN CUBIC BN-SURFACES}

We believe that $\mathrm{BN}$ crystal is very hard and is expected to yield very low friction coefficient in the dry sliding [47]. In view of what we learned from hydrogenated diamond surfaces, we next investigate atomic-scale friction between two cubic BN-slabs as shown in Figure 4.6(a) using the computational approach discussed above. These two slab are placed in a supercell with lattice constant $c=24 \AA$. Each slab consists of 5 atomic (100) planes. Atoms of last two layers are fixed in their equilibrium bulk positions but otherwise relax all the atomic degrees of freedom using a temperature annealing minimization as a function of slab-slab distance, $D$ and translation (along $x$-direction). In order to study the effect of the hydrogenation of the layers on the friction coefficient, we also consider two different hydrogen-saturated BN-surfaces as shown in Figure 4.6(b)-(c). For each system we have performed about 300 calculations, yielding almost thousand different configurations for the three systems shown in Figure 4.6.

(a) $\mathrm{BN}$

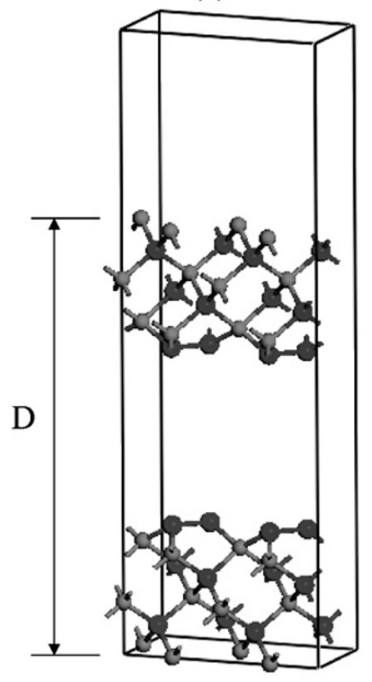

(b) $\mathrm{BNH}$

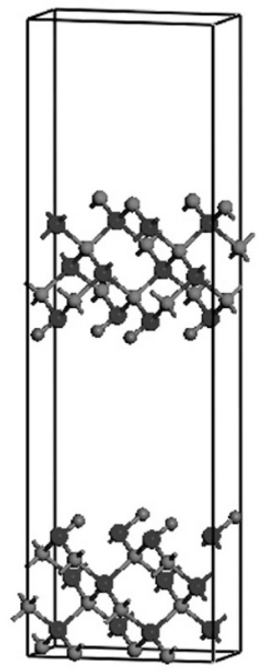

(c) $\mathrm{BNH}_{2}$

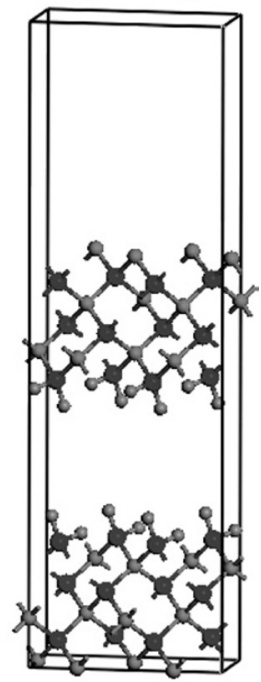

Figure 4.6 Three different slab structures that we considered in this study. (a) BN layers, which forms N-N-dimer on the surface. (b) Single hydrogen-passivated BNH slab with bended NH-bonds. (c) Doubly passivated $\mathrm{BNH}_{2}$ slab. The distance between the fixed-B atoms on the top and bottom slabs is shown as " $D$ ". The relevant bond-distances and atomic charges for these three structures are summarized in Table 4.1. 
Table 4.1.

Chemical formula, bond distances (in $\AA$ ), and Mulliken atomic charges (in electrons, e) for three systems shown in Figure 4.6. The lattice parameters of the supercell are $7.23 \times 7.23 \times 24(\AA)$ for all three systems. Below $Q_{\mathrm{s}}$ and $Q_{\mathrm{b}}$ indicates the atomic charges for atoms at the consecutive layers, respectively

\begin{tabular}{llll}
\hline System & Formula & Bond distances $(\AA)$ & Charges $(\mathrm{e})$ \\
\hline $\mathrm{BN}$ & $\mathrm{B}_{48} \mathrm{~N}_{48}$ & $d_{\mathrm{NN}}=1.42, d_{\mathrm{NB}}=1.56$ & $Q_{1}(\mathrm{~N})-1 / 3, Q_{2}(\mathrm{~N})=-2 / 3, \mathrm{Q}_{3}(\mathrm{~N})=-2 / 3$, \\
& & & $Q_{1}(\mathrm{~B})=1 / 3, Q_{2}(\mathrm{~B})=2 / 3, Q_{3}(\mathrm{~B})=2 / 3$ \\
$\mathrm{BNH}$ & $\mathrm{B}_{48} \mathrm{~N}_{48} \mathrm{H}_{16}$ & $d_{\mathrm{NH}}=1.02, d_{\mathrm{HH}}=2.56$, & $Q(\mathrm{H})=0.38$ \\
& & $d_{\mathrm{NN}}=2.56$ & $Q_{1}(\mathrm{~N})=-0.78, Q_{2}(\mathrm{~N})=-0.63, Q_{3}(\mathrm{~N})=-0.66$ \\
& & & $Q_{1}(\mathrm{~B})=0.71, Q_{2}(\mathrm{~B})=0.64, Q_{3}(\mathrm{~B})=0.35$ \\
$\mathrm{BNH}_{2}$ & $\mathrm{~B}_{48} \mathrm{~N}_{48} \mathrm{H}_{32}$ & $d_{\mathrm{HH}}=1.43,2.61$, & $Q(\mathrm{H})=0.25,0.39$ \\
& & $d_{\mathrm{NH}}=1.02,1.08$, & $Q_{1}(\mathrm{~N})=-0.91, Q_{2}(\mathrm{~N})=-0.65, Q_{3}(\mathrm{~N})=-0.65$ \\
& & $d_{\mathrm{NN}}=2.56$ & $Q_{1}(\mathrm{~B})=0.66, Q_{2}(\mathrm{~B})=0.62, Q_{3}(\mathrm{~B})=0.29$ \\
\hline
\end{tabular}

We first studied the total energy as a function of slab-slab distance as shown in Figure 4.7. For BN-layer, when the slabs are far enough, the energy is nearly zero and then goes to a minimum and then increases, with a attractive energy of $0.6 \mathrm{eV}$ (Figure 4.7(a)). The $\mathrm{N}$ atoms on the surface are negatively charged by $Q=-0.33$ electrons owing to the ionic nature of $\mathrm{BN}$ compound. The charge on the bulk $\mathrm{N}$ atoms are even larger. It is noticed that when two slabs is put close enough, the $\mathrm{N}$ atoms on different slabs are bonded to each other, resulting sudden decrease in the energy. However this adhesive behavior requires about $1 \mathrm{eV}$ energy barrier. Figure 4.7(b) shows the effect of the single hydrogenated BN-layer on the binding energy of the slabs. The energy curve is very different than that of bare BN layer shown in Figure 4.6(a). It has almost no minimum and mainly repulsive. It should be noted that GGA underestimates some of the vdW attraction. In principle, the interaction between two slabs should become slightly attractive when the Van der Waals interaction were included. This suggests that single hydrogenation of $\mathrm{N}$ atoms on the surface is expected to reduce the friction significantly and yield superlow friction coefficient. During the structural optimization of the BNH-layers, we also found a second metastable minima where the $\mathrm{H}$-atoms are attached to $\mathrm{B}$ atoms and aligned perpendicular to the surface. However the system lowers its energy by about $1.4 \mathrm{eV} /$ bond when the NH-bonds are bent so that BN-bond is parallel to the BN bonds as in the bulk (Figure 4.6(b)). Finally Figure 4.7(c) shows what happens if we saturate the $\mathrm{N}$ atom on the surface with an additional hydrogen atom. The energy curve looks like the bare BN layer but slightly larger binding energy of $0.9 \mathrm{eV}$. Hence, saturating with more hydrogen atom does not automatically mean that we would minimize the attractive part of the potential and make it repulsive. We anticipate that the large binding energy for the $\mathrm{BNH}_{2}$ case is mainly due to large atomic charges and thus the Coulomb interaction between the two slabs.

Finally, while we study the different ( $D$ or $z$ and $x$ ) slab configurations for $\mathrm{BNH}_{2}$, we observed that when the top slab moves along $x$-axis by about the half of the lattice constant at small slab-slab distances, two hydrogen atoms on the different slabs actually are bonded to each other forming a $\mathrm{H}_{2}$ molecule. The energy curve of the minimization as this process occurs is shown in Figure 4.8 along with the final system configuration where we have now one free $\mathrm{H}_{2}$ molecule. Hence for $\mathrm{BNH}_{2}$ surface, we expect reconstruction on the surface 

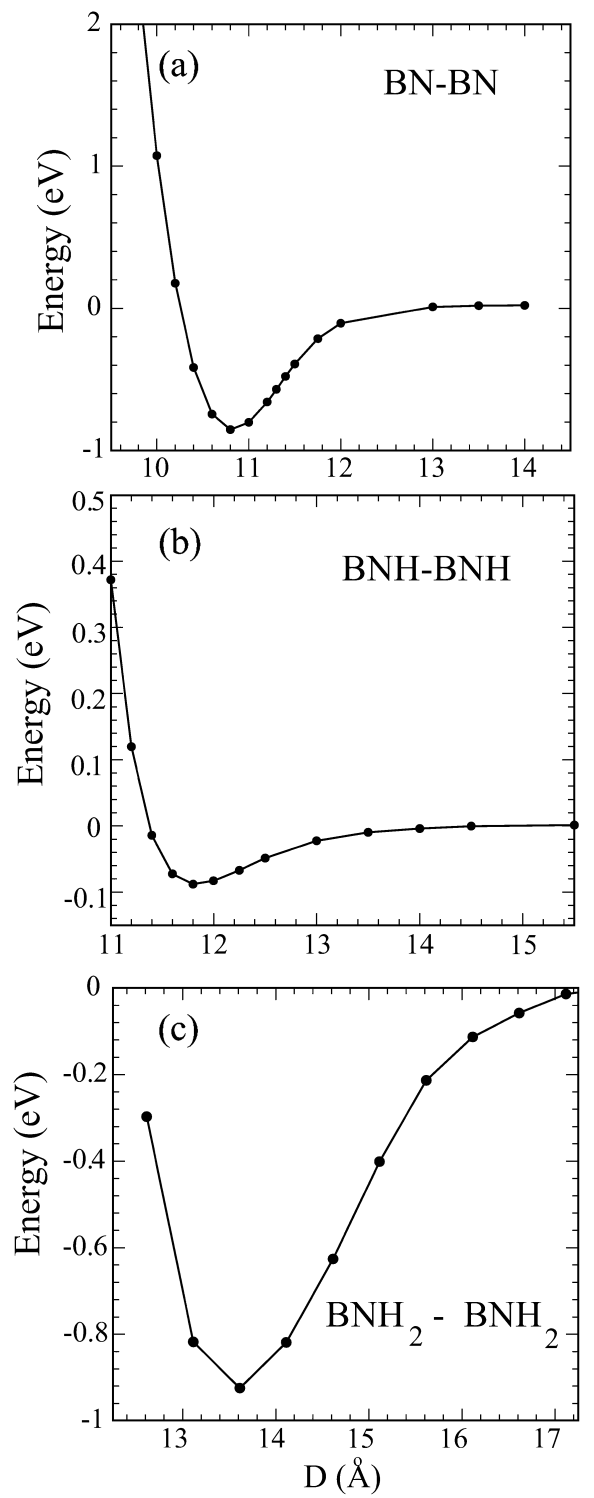

Figure 4.7 Energy curves as the slab-slab distance $D$ is varied for (a) $\mathrm{BN}$, (b) $\mathrm{BNH}$, (c) $\mathrm{BNH}_{2}$ slabs, respectively. Note that for $\mathrm{BNH}$ system, the potential curve is very flat and mainly repulsive. For $\mathrm{BNH}_{2}-\mathrm{BNH}_{2}$ we observed $\mathrm{H}_{2}$ formation from two hydrogen atoms of two slabs for $D<9.0 \AA$. This reconstruction of the slab surface is shown in Figure 4.8.

as the slabs move with respect to each other. When the slab-slab distance, $D$, is small enough, we also observe similar reconstructions even for bare BN-slabs as we discuss it briefly below. 


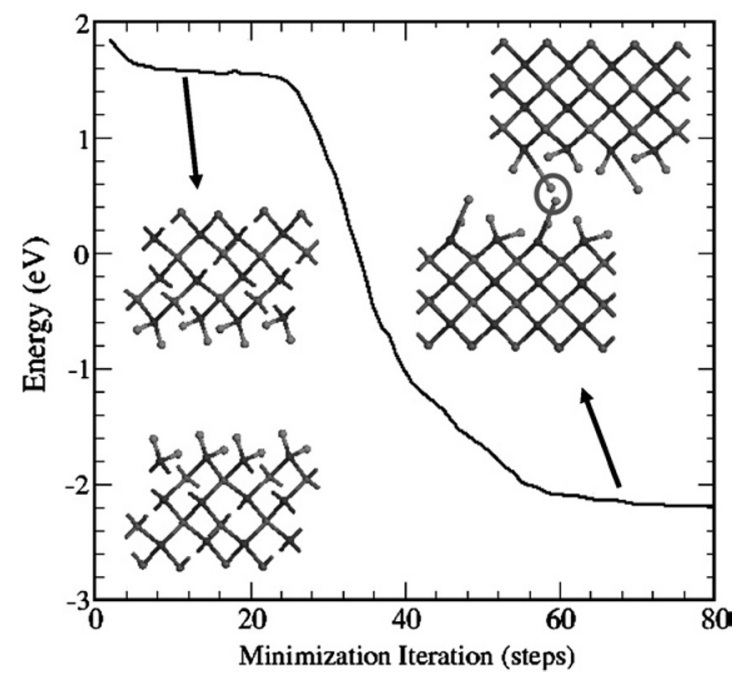

Figure 4.8 Energy curve during the structural minimization. When two slabs are close enough, the system lowers its energy by forming a free $\mathrm{H}_{2}$ molecule as the top slab moves along $x$-direction.

Figure 4.9 summarizes the energy and $z$-component of the total force (i.e. normal force) for the BN system for different values of slab-slab distance $D$ as the top slab is translated along the $x$-axis. For most of the slab-slab distances (i.e. $D=10.6-11.0 \AA$ ), the energy first increases, reaching its maximum at half-way $\Delta x$-translation and then decreases back. When the top slab is translated by one-lattice vector, we recover the original structure back, indicating that we are at the elastic regime. However this is not the case for $D=10.4 \AA$ or smaller slab-distances. Interestingly for $D=10.4 \AA$, the energy start to increase normally as the top-slab is being translated up to $\Delta x / a=0.5$ ( $a$ is the lattice constant of the surface unit cell along $x$-direction) but then the energy curves does not go back to its original value; instead continue to increase. This behavior becomes clear when we inspect the optimized atomic positions as the slabs are being translated along $x$-axis. We find that at $D=10.4 \AA$, the slab-slab interaction causes the $\mathrm{N}-\mathrm{B}$ bond on the surface to break, increasing it from $1.56 \AA$ to $1.75 \AA$. This is shown in the inset to Figure 4.9. In fact, for slab-slab distances less than $D=10.4 \AA$, we also observed that the broken $\mathrm{N}$-atom actually forms a new bond on the other $\mathrm{N}$-atom of the other slab. Hence we expect to see adhesive behavior between two BN-slabs when they are put together close enough. We note that the optimum distance between two BN-slabs is about $10.8 \AA$ (see Figure 4.6). Hence it is quite interesting that at $D=10.4 \AA$ (which is not too much different than $10.8 \AA$ ), there are already significant surface reconstructions. Figure 4.9(b) shows the normal forces acting on the BN slabs for different slab-slab distance. It shows an interesting variation; becomes almost flat with respect to slab translation at $z=10.7 \AA$. Since the $F_{z}$ is almost constant at this particular $z$, it is very convenient to calculate the atomic-scale friction using this particular slab-slab distance. We will discuss this further later.

Figure 4.10 summaries the energy and force curves for the BNH slab. The nature of $\mathrm{BNH}-\mathrm{BNH}$ slab interaction is very different than BN-slabs. This is clear by comparing the 

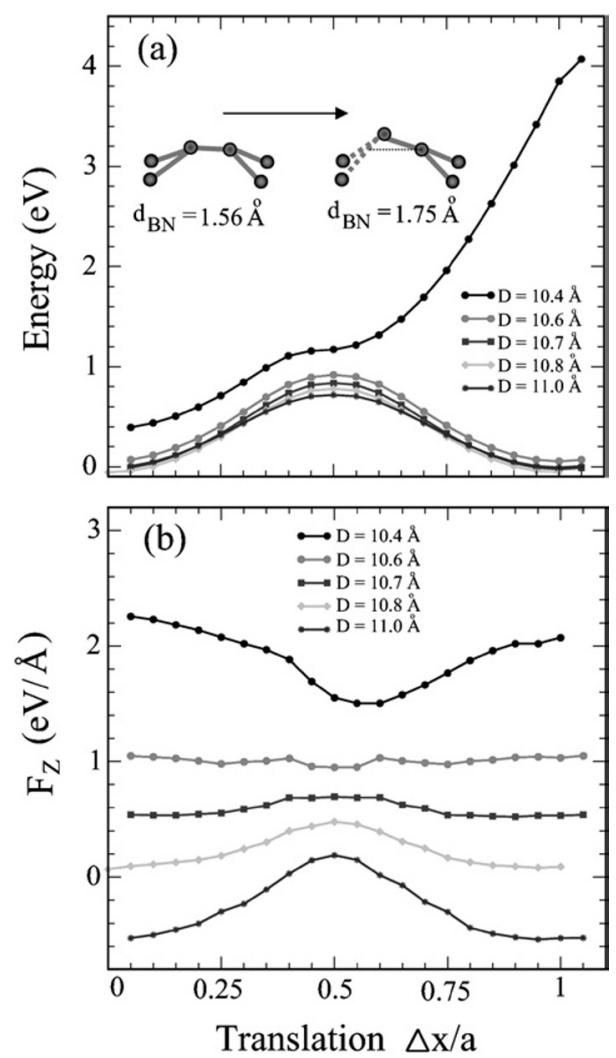

Figure 4.9 Energy and normal forces $F_{z}$ as the top slab is translated along $x$-direction with respect to bottom slab for different slab-slab distance $D$. For $D=10.4 \AA$, we observe significant surface reconstructions involving broken BN-bonds as depicted in inset to top panel.

curves shown in Figure 4.9 and Figure 4.10. We note that $F_{z}$ force acting on the BNH layer does not become constant for any slab-distance that we studied (unlike the BN case). The energies shown in Figure 4.10(a) are much smaller indicating the weak slab-slab interactions. Below we will combine the data presented in Figure 4.10 to obtain roughly constant force loading and estimate the atomic friction force.

As a last point, in Figure 4.11 we show some example plots of the forces on the slab as one of the slab is translated along $x$-direction for the three cases. We note that the resulting forces are oscillatory function of $\Delta x$-displacement for BN layer while it is very noisy for $\mathrm{BNH}_{2}$ layer. This is a nice demonstration of the effect of the surface structure on the energy and forces. In the case of BN layers, the surfaces are identical and in phase (or commensurate) with respect to each other. Therefore when one surface slides we see large variations in the energy and forces. However for the case of $\mathrm{BNH}_{2}$ system, the surface structure is quite complicated due to four $\mathrm{H}$ atoms and therefore two surfaces almost random (incommensurate) with respect to each other. Hence when we translate one slab, it 

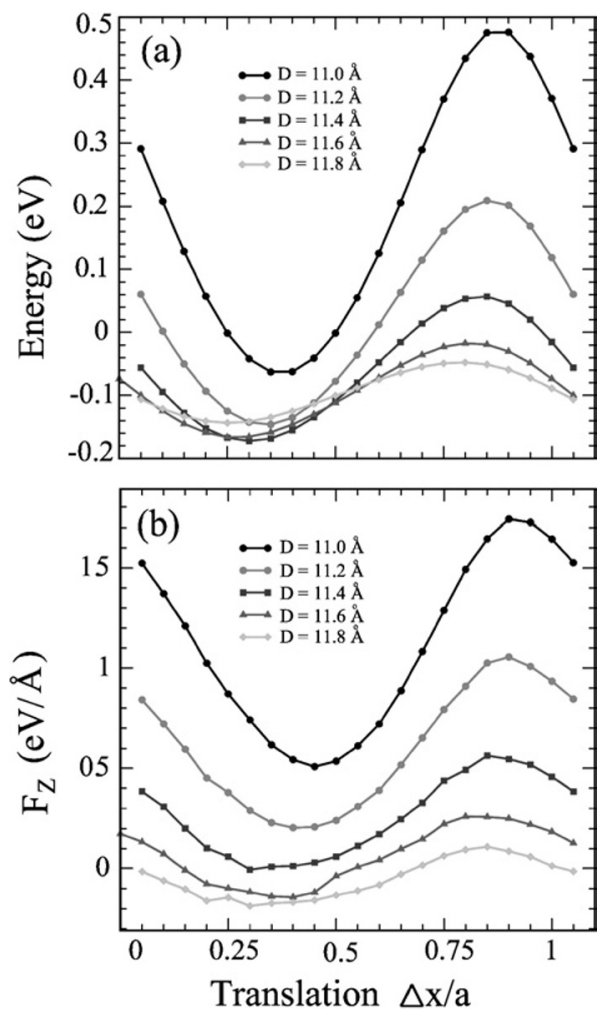

Figure 4.10 (a) Energy and (b) normal forces $F_{z}$ as the top BNH slab is translated along $x$-direction with respect to bottom slab for different slab-slab distance $D$.

does not change the energy or the forces with respect to each other. This confirms the fact that the friction coefficient is usually low between incommensurate surfaces.

Finally we obtained an estimate for the friction coefficients using the forces shown in Figure 4.11. For the normal loading force, we integrate the $F_{z}$ over the cell to obtain an average force. For the $x$ - and $y$-components of the lateral force, if we integrate them over the cell, we always obtain very small number from 0.01 to $0.001 \mathrm{eV} / \AA$, suggesting that the most of the relaxation process during the minimization is in the elastic range. If one assumes that the induced strain or energy is efficiently removed from the system by phonons, then we can estimate an upper limit for the friction. In compliance with the discussion in Section 4.4.2, for this purpose, we calculate the average value of the lateral force $F_{x}$ which is in opposite direction of the displacement. The average values are given in Figure 4.11. We note that by hydrogenating the BN layers, we reduce the friction coefficient significantly (which is given by $\mu_{k}=F_{x} / F_{z}=0.06$ ). $\mathrm{BNH}_{2}$ layer has slightly lower friction coefficient $\left(\mu_{k}=0.03\right)$ than BN layer even though the energy dependence of the slab-slab interactions are very different. In the case of BN, it's mainly repulsive while in the case of $\mathrm{BNH}_{2}$ it has strong attractive component. However as we discussed above, the friction is 

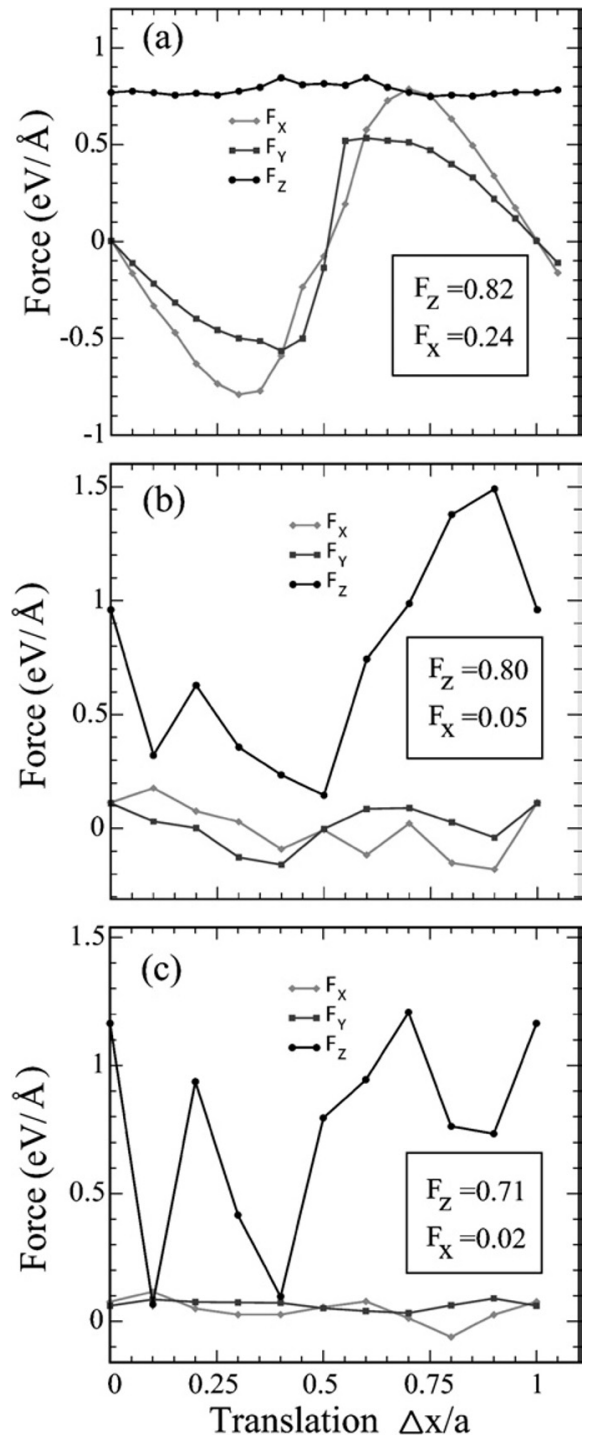

Figure 4.11 Forces as the top slab is translated along $x$-direction with respect to bottom slab. The values of the absolute-averaged forces over the unit cell are also given. For $F_{x}$ and $F_{y}$, the average forces are nearly zero (less than $0.01 \mathrm{eV} / \AA$ ). The numbers are for the integration of the absolute forces for (a) BN, (b) BNH, (c) $\mathrm{BNH}_{2}$ slabs, respectively.

very low in $\mathrm{BNH}_{2}$ because the incommensurability of the surfaces due to complex atomic structure of the surface.

The above atomic-scale study of dry sliding friction between bare and hydrogenated cubic BN (001) surfaces exhibit differences from that between two diamond $(001)-(2 \times 1)$ surfaces. While the interaction between bare BN (001) surfaces weakly attractive, it be- 
comes strongly repulsive upon $\mathrm{H}$-saturation of each $\mathrm{N}$ atom. On the other hand, the dihydride formation on $\mathrm{BN}$-layers by adsorbing two hydrogen atom to each $\mathrm{N}$ atom show strong attractive potential. However, the resulting forces and variation in the energy as the slabs are translated with respect to each other is still small. This is due to a different effect; namely the incommensurability of the surfaces with respect to each other. With two $\mathrm{H}$ atoms saturating $\mathrm{N}$ atoms, we obtain complicated surface structure and therefore as one slide moves with respect to other, nothing is really changed, resulting small forces and binding energy. Stated differently, even if $\left|F_{z}\right|$ is large, lateral force components, $F_{x}$ and $F_{y}$ may become small due to cancellations. We also observed that under very large loading forces (with activation energy of several $\mathrm{eV}$ ), it is possible to obtain surface reconstruction and even $\mathrm{H}_{2}$ formation by breaking the $\mathrm{NH}$ bonding.

\subsection{CONCLUSIONS}

We presented a comparative study of the dry sliding friction between the atomically flat and commensurate surfaces of two different systems, namely between two diamond (001) and between two BN (001) surfaces. While the interaction between bare diamond (001)$(2 \times 1)$ surfaces is strongly attractive until small spacing $d \sim 1.5 \AA$ and forms strong chemical bonds between two surfaces. However, the interaction turns repulsive if two surfaces are pushed under high loading force. Upon hydrogenation, hydrogen atoms donate charge to the carbon atom and become positively charged. Then the interaction between surfaces carrying the same type charge becomes repulsive. This appears to be the most important ingredient of the superlow friction. The repulsive interaction persists at any relative position of the sliding surfaces and is strong even at large distance to prevent $\mathrm{C}-\mathrm{H}$ bonds from merging. Strong and stiff $\mathrm{C}-\mathrm{H}$ bonds and stiff diamond crystal by itself prevent excessive energy from dissipation. It is found that oxygenation of surfaces in the atmospheric conditions destroy the steady repulsive interaction.

However the interaction between two bare reconstructed BN (001) surfaces are different from that of the diamond (001) surface due to ionic nature of the crystal. BN being an ionic crystal with electron transfer from $\mathrm{B}$ to $\mathrm{N}$ the bare surface is already negatively charged. Under these circumstances the strong chemical interaction is canceled by the repulsive Coulombic interaction resulting in a weak attractive interaction (or adhesive forces). As a result, the friction coefficient is already small in dry sliding friction two bare BN (001) surfaces. Upon saturation of each $\mathrm{N}$ atom by a single $\mathrm{H}$-atom the weak attractive interaction is further reduced. This situation changes when single $\mathrm{N}$ atom is saturated by two $\mathrm{H}$ atoms, and the interaction becomes again attractive. The present analysis of dry sliding friction between bare and hydrogenated $\mathrm{BN}(001)$ surfaces indicate that this material is a potential candidate for superlow friction.

\section{ACKNOWLEDGEMENTS}

This work was supported by Scientific and Technological Council of Turkey, TÜBİTAK under Grant No. TBAG-104T537. Authors acknowledge the useful assistance of Engin Durgun in preparation of the manuscript. 


\section{REFERENCES}

[1] Bowden, F.P., Tabor, D. Friction and Lubrication. Methuen, London, 1965.

[2] Rabinowitz, E. Friction and Wear. Wiley, New York, 1965.

[3] Persson, B.N.J. Sliding Friction: Physical Principles and Applications. Springer-Verlag, Berlin, 2000.

[4] Israelachvili, J.N. Intermolecular and Surface Forces. Academic Press, London, 1985.

[5] Ciraci, S., Baratoff, A., Batra, I.P. Phys. Rev. B 42 (1990), 7168; Ciraci, S., Tekman, E., Baratoff, A., Batra, I.P. Phys. Rev. B 46 (1992), 10411.

[6] Buldum, A., Ciraci, S. Phys. Rev. B 55 (1997), 12892.

[7] Buldum, A., Ciraci, S. Phys. Rev. B 55 (1997), 2606.

[8] Tomlinson, G.A. Philos. Mag. 7 (1929), 905.

[9] Frenkel, J., Kontorova, T. Phys. Z. Sowjet 13 (1938), 1.

[10] Binnig, G., Quate, C.F., Gerber, Ch. Phys. Rev. Lett. 56 (1986), 960.

[11] Mate, C.M., McClelland, G.M., Erlandsson, R., Chiang, S. Phys. Rev. Lett. 59 (1987), 1942.

[12] Meyer, E., Overney, R., et al. Phys. Rev. Lett. 69 (1992), 1777.

[13] Sutton, A.P., Pethica, J.B. J. Phys.: Condens. Matter 2 (1990), 5317.

[14] Cieplak, M., Smith, E.D., Robins, M.O. Science 265 (1994), 1209.

[15] Bhushan, B., Israelachivili, J.N., Landman, U. Nature 347 (1995), 607.

[16] Sorensen, M.R., Jacobsen, K.W., Stoltze, P. Phys. Rev. B 53 (1996), 2101.

[17] Buldum, A., Ciraci, S. Phys. Rev. B 57 (1998), 2468.

[18] Buldum, A., Ciraci, S. Phys. Rev. B 60 (1999), 1982.

[19] Hohenberg, P., Kohn, W. Phys. Rev. B 136 (1964), B864; Kohn, W., Sham, L.J. Phys. Rev. 140 (1965), A1133.

[20] Zhong, W., Tomanek, D. Phys. Rev. Lett. 64 (1990), 3054; Tomanek, D., Zhong, W., Thomas, H. Europhys. Lett. 15 (1991), 887.

[21] Buldum, A., Leitner, D.M., Ciraci, S. Europhys. Lett. 47 (1999), 208.

[22] Buldum, A., Leitner, D.M., Ciraci, S. Phys. Rev. B 59 (1999), 16042.

[23] Ozpineci, A., Leitner, D.M., Ciraci, S. Phys. Rev. B 62 (2000), 10558.

[24] Ozpineci, A., Ciraci, S. Phys. Rev. B 63 (2001), 125415.

[25] Sokoloff, J.B. Phys. Lett. 71 (1993), 3450.

[26] Giessibl, F.J. Science 267 (1995), 68.

[27] Bammerlin, M., Lüthi, R., Meyer, E., Baratoff, A., Lu, J., Guggisberg, M., Gerber, Ch., Howald, L., Güntherodt, H.-J. Probe Microscopy 1 (1997), 3.

[28] Gauthier, M., Tsukada, M. Phys. Rev. B 60 (1999), 11716.

[29] Guggisberg, M., Bammerlin, M., Lüthi, R., Loppacher, Ch., Battiston, F., Lu, J., Baratoff, A., Meyer, E., Güntherodt, H.-J. Appl. Phys. A 66 (1998), S245.

[30] Kantorovich, L.N. Phys. Rev. B 64 (2001), 245409.

[31] Martin, J.M., Donnet, C., Le Mogne, Th., Epicier, Th. Phys. Rev. B 48 (1993), 10583.

[32] Sugimoto, I., Miyake, S. Appl. Phys. Lett. 56 (1990), 1868.

[33] Donnet, C., Grill, A. Surf. Coat. Technol. 94-95 (1997), 456.

[34] Erdemir, A., Eryilmaz, O.L., Fenske, G. J. Vac. Sci. Technol. A 18 (2000), 1987.

[35] Erdemir, A. Surf. Coat. Technol. 146-147 (2001), 292.

[36] Heimberg, J.A., Wahl, K.J., Singer, I.L., Erdemir, A. Apl. Phys. Lett. 78 (2001), 2449.

[37] Vanderbilt, D. Phys. Rev. B 41 (1990), 7892.

[38] Numerical calculations have been performed by using VASP package: Kresse, G. Hafner, J. Phys. Rev. B 47 (1993), 558; Kress, G., Furthmüller, J., ibid 54 (1996), 11169.

[39] Perdew, J.P., Chevary, J.A., Vosko, S.H., Jackso, K.A., Pederson, M.R., Singh, D.J., Fiolhais, C. Phys. Rev. B 46 (1992), 6671.

[40] Monkhorst, H.J., Pack, J.D. Phys. Rev. B 13 (1976), 5188.

[41] Dag, S., Ciraci, S. Phys. Rev. B 70 (2004), 241401.

[42] Gao, G.T., Mikulski, P.T., Harrison, J.A. J. Am. Chem. Soc. 124 (2002), 7202; Gao, G.T., Mikulski, P.T., Chateauneuf, G.M., Harrison, J.A. J. Phys. Chem. B 107 (2003), 11082.

[43] Levitov, L.S. Europhys. Lett. 8 (1989), 449. 
[44] Persson, B.N.J. Phys. Rev. B 44 (1991), 3227; Persson, B.N.J., Volotkin, A.I. J. Chem. Phys. 103 (1995), 8679.

[45] Dayo, A., Alnasrallah, W., Krim, J. Phys. Rev. Lett. 80 (1998), 1690.

[46] Thoms, B.D., Butler, J.E. Phys. Rev. B 50 (2000), 17450.

[47] Miyoshi, K., Buckly, D.H., Spalvins, T. J. Vac. Sci. Technol. A 3 (1985), 2340. 